\title{
Correction to: Reducing surgical site infections following total hip and knee arthroplasty: an Israeli experience
}

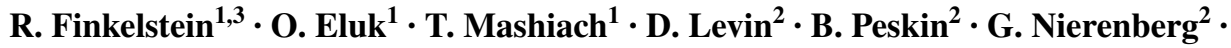 \\ S. Karkabi ${ }^{2} \cdot$ M. Soudri ${ }^{2,3}$
}

Published online: 12 October 2017

(C) Istituto Ortopedico Rizzoli 2017

\section{Correction to: Musculoskelet Surg \\ DOI 10.1007/s12306-017-0471-2}

In the original article, one of the co-author's family name has been published incorrectly.

The correct family name should be Nierenberg.

The online version of the original article can be found under doi:10.1007/s12306-017-0471-2.

R. Finkelstein

renatof@bezeqint.net

1 Infectious Diseases Unit, Rambam Medical Center, Rechov Alia Shnia, 31096 Haifa, Israel

2 Department of Orthopaedic Surgery, Rambam Medical Center, 31096 Haifa, Israel

3 The Bruce Rappaport Faculty of Medicine, Technion, Israel Institute of Technology, Haifa, Israel 